\title{
Synthesis of conformationally constrained, orthogonally protected 3-azabicyclo[3.2.1]octane $\beta$-amino esters
}

\author{
Brigitta Kazi, ${ }^{a}$ Loránd Kiss, ${ }^{a}$ Enikő Forró, ${ }^{a}$ István Mándity, ${ }^{a}$ and Ferenc Fülöp ${ }^{\text {a,b* }}$ \\ ${ }^{a}$ Institute of Pharmaceutical Chemistry, ${ }^{b}$ Stereochemistry Research Group of the Hungarian \\ Academy of Sciences, University of Szeged, H-6720 Szeged, Eötvös u. 6, Hungary \\ E-mail: fulop@pharm.u-szeged.hu
}

Dedicated to the memory of Professor Gábor Bernáth (1933-2009)

\begin{abstract}
Novel azabicyclic $\beta$-amino acid derivatives were readily prepared from diexo or diendo norbornene $\beta$-amino acids. The 3-azabicyclo[3.2.1] octane skeleton was obtained by $\mathrm{NaIO}_{4}$ mediated cleavage of the dihydroxylated $\beta$-amino ester intermediates, followed by reductive amination. Lipase-catalyzed enantioselective ring opening of racemic exo-norbornene $\beta$-lactam allowed preparation of the corresponding azabicyclic exo $\beta$-amino acid in enantiopure form.
\end{abstract}

Keywords: $\beta$-amino acids, dihydroxylation, ring opening, ring closure, enzymatic resolution

\section{Introduction}

Because of their importance in synthetic and medicinal chemistry and in peptide research, conformationally rigid, alicyclic $\beta$-amino acids have been subject to considerable interest during the past 20 years $^{1}$. $N$-Heterocyclic $\beta$-amino acids have also attracted attention in view of their biological properties and their applications in peptide synthesis. ${ }^{2}$ Bicyclic $\alpha$ - or $\beta$-amino acids in which the $N$ atom of the amino function is part of the ring system are a class of compounds of appreciable importance. Thus, bicyclic $\alpha$-amino acids with the $\mathrm{N}$ atom in the ring system, such as 7-azabicyclo[2.2.1] heptane-1-carboxylic acid, its derivatives and compounds with an 8azabicyclo[3.2.1]octane skeleton are conformationally restricted analogs of proline, hydroxyprolines and related proline derivatives. ${ }^{3,4}$ 7-Azabicyclo[2.2.1] heptane-2-carboxylic acid $\beta$-amino acids key compounds in novel $\beta$-peptide syntheses, ${ }^{5}$ were recently reported to behave as conformationally restricted proline analogs, acting as efficient catalysts in organocatalytic aldol processes $^{6}$ Moreover, both bicyclic $\alpha$ - and $\beta$-amino acids with the $N$ atom in the ring system serve as key precursors for the synthesis of medicinally valuable alkaloids such as anatoxin- $a,{ }^{7}$ epibatidine, epiboxidine etc. ${ }^{8}$ A number of pharmacologically active 3-azabicyclo[3.2.1] octanes 
have been reported as bioactive molecules, ${ }^{9}$ the most important of them probably being those with an amino or carboxyl function in their structure. 3-Azabicyclo[3.2.1] octane $\alpha$-amino acids were recently synthetized in enantiomerically pure form, ${ }^{9 a}$ Because of the importance of the conformationally constrained alicyclic or heterocyclic $\beta$-amino acids, our work was directed toward the synthesis of novel 3-azabicyclo[3.2.1] octane skeleton $\beta$-amino acids.

\section{Results and Discussion}

The synthetic route applied for the preparation of these azabicyclic $\beta$-amino esters is based on a simple method used for the synthesis of piperidine and azepane $\beta$-amino carboxylates. ${ }^{2 \mathrm{a}, \mathrm{b}}$ The starting compounds were exo and endo norbornene $\beta$-amino acids. ${ }^{10}$ Diexo- $N$-Boc-protected norbornene amino ester 1 was transformed into the corresponding dihydroxy derivative $\mathbf{2}$ with $\mathrm{N}$ methylmorpholine- $N$-oxide (NMO) as stoichiometric co-oxidant and $\mathrm{OsO}_{4}$ as catalyst. A single dihydroxylated amino ester diastereomer was selectively obtained, in which the cis situation of the hydroxy groups relative to the methylene bridge was confirmed by NOESY experiment. NOE signals were observed between OH-5 ( $\delta$ 4.63), OH-6 $(\delta 4.63)$ and H-7 $(\delta 1.81)$. Oxidative breaking of the vicinal diol $\mathrm{C}-\mathrm{C}$ bond in 2 with $\mathrm{NaIO}_{4}$ gave the corresponding dialdehyde. As this is unstable, its solution was submitted immediately, without isolation, to reductive amination with benzylamine in the presence of $\mathrm{NaBH}_{3} \mathrm{CN}$ and $\mathrm{AcOH}$. The ring-closure reaction afforded the desired azabicyclic $\beta$-amino ester 3 (Scheme 1).

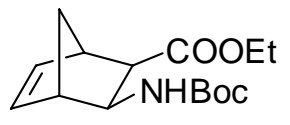

1

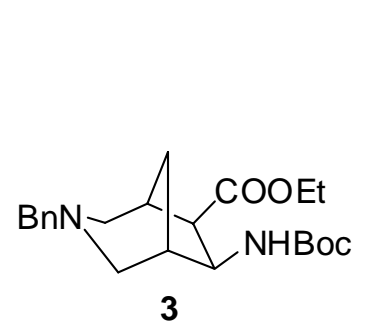

3

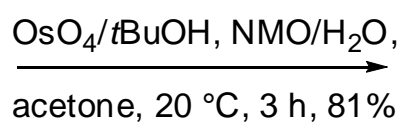

acetone, $20^{\circ} \mathrm{C}, 3 \mathrm{~h}, 81 \%$

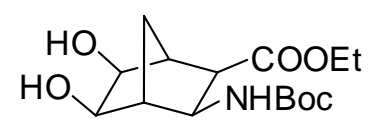

2

$\mathrm{NaIO}_{4}$ $\mathrm{THF} / \mathrm{H}_{2} \mathrm{O}$, $\checkmark 20{ }^{\circ} \mathrm{C}, 1 \mathrm{~h}$

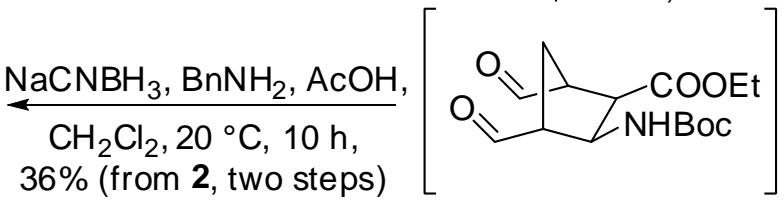

Scheme 1. Syntheses of racemic azabicyclic $\beta$-amino ester $\mathbf{3}$.

The above synthetic route was extended to the preparation of $\beta$-amino ester $(+)-3$ in enantiomerically pure form (ee $>99 \%$ ) from $(+)-1$, synthetized by a literature method. ${ }^{11}$ Racemic norbornene $\beta$-lactam was subjected to enzymatic ring opening in the presence of CAL-B (lipase $\mathrm{B}$ from Candida antarctica, produced by the submerged fermentation of a genetically modified Aspergillus oryzae microorganism and adsorbed on a macroporous resin) in $\mathrm{iPr}_{2} \mathrm{O} .{ }^{11} \operatorname{The}$ 
unreacted $\beta$-lactam enantiomer was transformed into the protected amino ester $(+)-\mathbf{1}$ via lactam ring opening, followed by N-Boc protection. Further transformations (dihydroxylation, ringcleavage and ring-closure) were performed similarly as for the racemic substances, resulting in the enantiomerically pure bicyclic $\beta$-amino ester $(+)-3$ (Scheme 2 ).

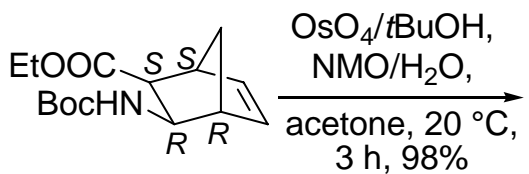

$(+)-1$

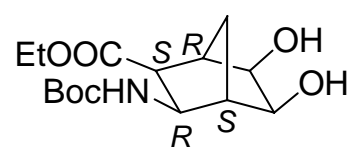

$(+)-2$
1. $\mathrm{NaIO}_{4}, \mathrm{THF} / \mathrm{H}_{2} \mathrm{O}$,

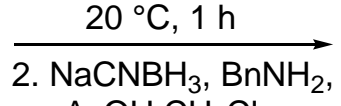
$\mathrm{AcOH}, \mathrm{CH}_{2} \mathrm{Cl}_{2}$, $20{ }^{\circ} \mathrm{C}, 10 \mathrm{~h}, 38 \%$

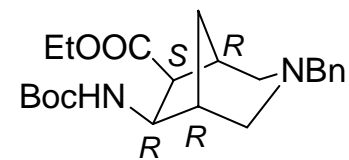

$(+)-3$

Scheme 2. Synthesis of enantiomeric azabicyclic $\beta$-amino ester (+)-3.

Via a synthetic procedure similar to that presented in Scheme 1, starting from diendo norbornene amino ester 4 through dihydroxylation, cleavage of the resulting diol and reductive amination two azabicyclic stereoisoimers $\mathbf{6}$ and the earlier synthetized $\mathbf{3}$ were obtained in a ratio of 9:1 (Scheme 3). The NMR data confirmed that the major product was the desired heterocyclic diendo amino ester $\mathbf{6}$, while the minor product was identified as the diexo compound $\mathbf{3}$ (Scheme $3)$.

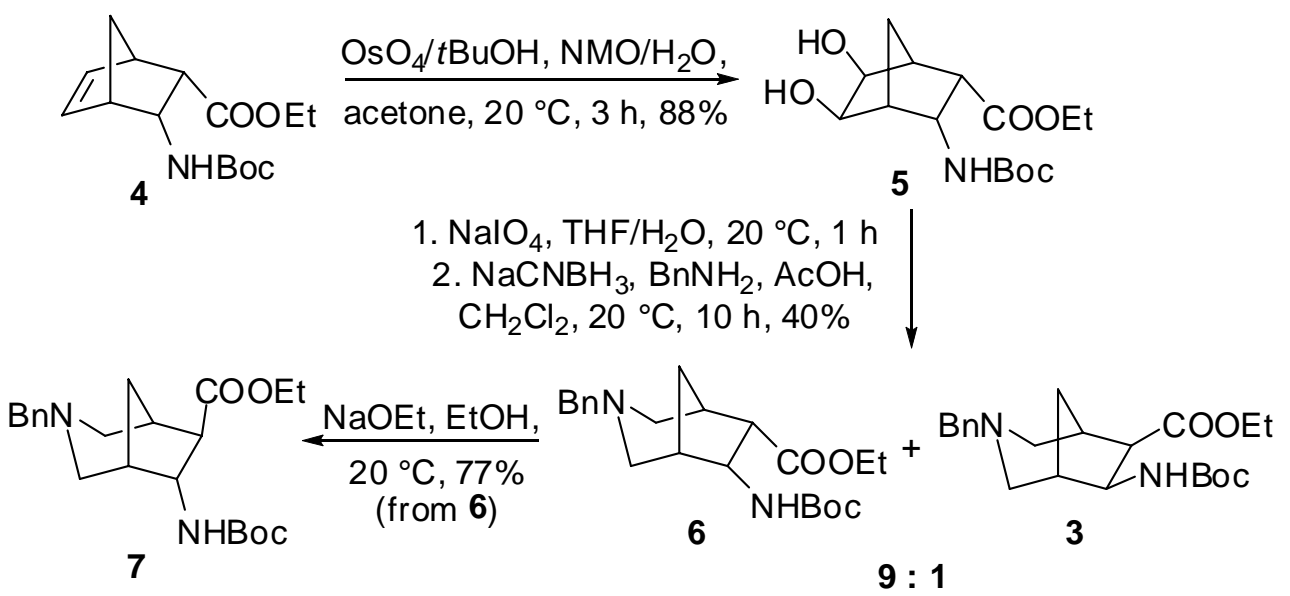

Scheme 3. Syntheses of azabicyclic $\beta$-amino esters 3,6 and 7 .

The detection of $\mathbf{3}$ in this reaction mixture was somewhat surprising as it demands a simultaneous configuration change at two positions (Scheme 3). The explanation may be that the keto-enol tautomerism of the dialdehyde intermediate generated from the oxidative cleavage of diol 5, results in the configurational change of the heterocyclic amino ester (diendo-diexo), thereby accounting for the mixture of ring closure products (Scheme 4). The heterocyclic diendo amino ester $\mathbf{6}$ was subjected to isomerization in the presence of $\mathrm{NaOEt}$ in EtOH to afford a new 
azabicyclic diastereomer 7, a C-2 epimer of 6, in good yield (77\%) with almost full conversion (Scheme 3).

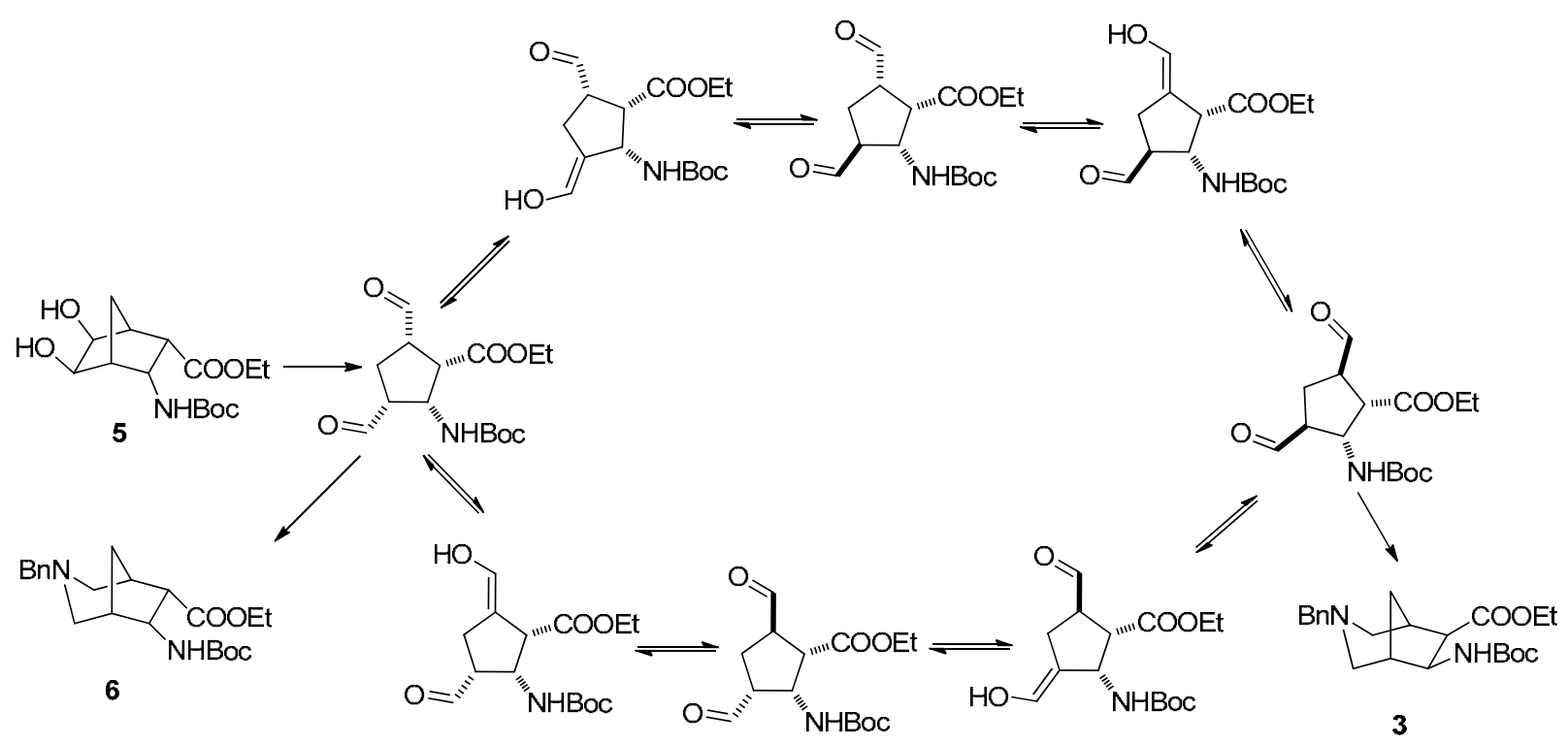

Scheme 4. Possible stereoisomers of the dialdehyde intermediate due to keto-enol tautomerism.

\section{Experimental Section}

General. The chemicals were purchased from Aldrich or Fluka. The solvents were used as received from the supplier. NMR spectra were recorded on a Bruker DRX 400 spectrometer. Chemical shifts are given in $\delta(\mathrm{ppm})$. Optical rotations were measured with a Perkin-Elmer 341 polarimeter. Melting points were determined with a Kofler apparatus. The mass spectra were recorded on a Finnigan MAT 95S spectrometer. Elemental analyses were performed with a Perkin-Elmer CHNS-2400 Ser II Elemental Analyzer. The ee value for (+)-3 was determined by HPLC on a Chiral Pak IA $5 \mu$ column $(0.4 \mathrm{~cm} \mathrm{x} 1 \mathrm{~cm})$ [mobile phase: $n$-hexane/2-propanol (90/10); flow rate $0.5 \mathrm{~mL} / \mathrm{min}$; detection at $205 \mathrm{~nm}$; retention time (min): 16.6 (antipode: 12.7)].

\section{General procedure for Boc protection of amino esters}

To a solution of ethyl diexo- or diendo-3-aminobicyclo[2.2.1]hept-5-ene-2-carboxylate hydrochloride $^{10}(2 \mathrm{~g}, 9.23 \mathrm{mmol})$ in THF $(40 \mathrm{~mL}), \mathrm{Et}_{3} \mathrm{~N}(1.86 \mathrm{~g}, 18.4 \mathrm{mmol})$ and di-tert-butyl dicarbonate $(2.21 \mathrm{~g}, 10.1 \mathrm{mmol})$ were added at $0{ }^{\circ} \mathrm{C}$. After stirring for $20 \mathrm{~h}$ at room temperature, the reaction mixture was taken up in EtOAc $(40 \mathrm{~mL})$ and the solution was washed with $\mathrm{H}_{2} \mathrm{O}(3 \times$ $80 \mathrm{~mL}$ ), dried over $\mathrm{Na}_{2} \mathrm{SO}_{4}$ and concentrated under reduced pressure, giving amino ester 1 or 4 .

Ethyl diexo-(1S*,2S*,3R*,4R*)-3-(tert-butoxycarbonylamino)bicyclo[2.2.1]hept-5-ene-2carboxylate (1). White crystals; $96 \%$ yield; mp. 141-143 ${ }^{\circ} \mathrm{C} .{ }^{1} \mathrm{H}$ NMR (DMSO, $\left.400 \mathrm{MHz}\right) \delta$ : 
1.17 (t, 3H, J=7.05 Hz, CH 3 ), 1.37 (s, 10H, tBu, H-7), 2.12 (d, 1H, H-7, J = 9.06 Hz), 2.45 (d, $1 \mathrm{H}, \mathrm{H}-2, J=8.05 \mathrm{~Hz}$ ), 2.54-2.60 (m, 1H, H-1), 2.83-2.87 (m, 1H, H-4), 3.77-3.81 (m, 1H, H-3), 3.89-4.00 (m, 1H, $\left.\mathrm{OCH}_{2}\right), 4.00-4.11\left(\mathrm{~m}, 1 \mathrm{H}, \mathrm{OCH}_{2}\right), 6.16-6.23(\mathrm{~m}, 2 \mathrm{H}, \mathrm{H}-5$ and H-6), 6.63 (d, $1 \mathrm{H}, \mathrm{NH}, J=8.81 \mathrm{~Hz}) .{ }^{13} \mathrm{C}$ NMR (DMSO, $400 \mathrm{MHz}$ ) $\delta: 14.9,27.7,29.1,45.1,45.9,48.1,54.0$, 60.6, 78.6, 137.9, 139.7, 155.8, 173.5. Anal. Calcd for $\mathrm{C}_{15} \mathrm{H}_{23} \mathrm{NO}_{4}$ : C, 64.03; H, 8.24; N, 4.98. Found: C, 63.91; H, 8.20; N, 4.97.

Ethyl diendo-(1 $\left.R^{*}, 2 S^{*}, 3 R^{*}, 4 S^{*}\right)$-3-(tert-butoxycarbonylamino)bicyclo[2.2.1]hept-5-ene-2carboxylate (4). White crystals; 83\% yield; m.p. 86-88 ${ }^{\circ} \mathrm{C} .{ }^{1} \mathrm{H}$ NMR (DMSO, $\left.400 \mathrm{MHz}\right) \delta: 1.15$ (t, 3H, $J=7.08 \mathrm{~Hz}, \mathrm{CH}_{3}$ ), 1.26-1.45 (m, 11H, tBu, H-7), 2.88-2.92 (m, 1H, H-1), 2.97-3.01 (m, 1H, H-4), 3.13-3.21 (m, 1H, H-2), 3.88-4.05 (m, 2H, $\left.\mathrm{OCH}_{2}\right), 4.38-4.49$ (m, 1H, H-3), $6.62(\mathrm{~d}$, $1 \mathrm{H}, \mathrm{NH}, J=8.81 \mathrm{~Hz}), 6.15-6.21(\mathrm{~m}, 1 \mathrm{H}, \mathrm{H}-6), 6.28-6.34(\mathrm{~m}, 1 \mathrm{H}, \mathrm{H}-5) .{ }^{13} \mathrm{C}$ NMR (DMSO, 400 MHz) $\delta: 14.9,29.0,46.5,47.5,47.8,49.3,54.5,60.5,78.8,134.0,138.7,155.7,172.7$. Anal. Calcd for $\mathrm{C}_{15} \mathrm{H}_{23} \mathrm{NO}_{4}$ : C, 64.03; H, 8.24; N, 4.98. Found: C, 64.00; H, 8.35; N, 5.11.

\section{General procedure for dihydroxylation of $\mathrm{N}$-Boc-protected amino esters}

$\mathrm{OsO}_{4}(1.43 \mathrm{~mL}, 0.08 \mathrm{mmol} ; 0.06 \mathrm{M}$ solution in $t \mathrm{BuOH})$ was added to a stirred solution of NMO (1.98 mL, $9.41 \mathrm{mmol} ; 50 \mathrm{wt} . \%$ in $\left.\mathrm{H}_{2} \mathrm{O}\right)$ and amino ester 1 or 4 (2.5 g, $\left.8.88 \mathrm{mmol}\right)$ in acetone (20 $\mathrm{mL})$. After $3 \mathrm{~h}$, the mixture was treated with saturated aqueous $\mathrm{Na}_{2} \mathrm{SO}_{3}(25 \mathrm{~mL})$ and filtered through Celite, and the Celite was washed with $\mathrm{CH}_{2} \mathrm{Cl}_{2}(60 \mathrm{~mL})$. The phases were separated and the organic layer was washed with saturated aqueous $\mathrm{NaHCO}_{3}(2 \times 25 \mathrm{~mL})$, dried over $\mathrm{Na}_{2} \mathrm{SO}_{4}$, and concentrated under reduced pressure to give the crude solid, which was recrystallized from $n$-hexane/EtOAc. The oily compound was purified by column chromatography on silica gel ( $n$ hexane/EtOAc, 1:3).

Ethyl diexo-(1R*,2S*,3R*,4S*)-3-(tert-butoxycarbonylamino)-5,6-dihydroxybicyclo[2.2.1]heptane-2carboxylate (2). White crystals; 81\% yield; m.p. 116-118 ${ }^{\circ} \mathrm{C} .{ }^{1} \mathrm{H}$ NMR (DMSO, $\left.400 \mathrm{MHz}\right) \delta: 1.14$ $\left(\mathrm{t}, 3 \mathrm{H}, J=7.08 \mathrm{~Hz}, \mathrm{CH}_{3}\right), 1.35(\mathrm{~s}, 9 \mathrm{H}, t \mathrm{Bu}), 1.61(\mathrm{~d}, 1 \mathrm{H}, \mathrm{H}-7, J=10.82 \mathrm{~Hz}), 1.75-1.83$ (m, 2H, H-7 and, H-1), 2.05-20.10 (m, 1H, H-4), 2.46-3.00 (m, 1H, H-2), 3.47-3.57 (m, 2H, H-5 and H6), 3.78-4.01 (m, 1H, H-3), 3.84-3.95 (m, 1H, OCH $), 3.96-4.08\left(\mathrm{~m}, 1 \mathrm{H}, \mathrm{OCH}_{2}\right), 4.63$ (brs, $2 \mathrm{H}$, $\mathrm{OH}), 6.62(\mathrm{~d}, 1 \mathrm{H}, \mathrm{NH}, J=8.81 \mathrm{~Hz}) .{ }^{13} \mathrm{C} \mathrm{NMR}(\mathrm{DMSO}, 400 \mathrm{MHz}) \delta: 14.8,29.0,29.9,46.5$, 49.2, 49.7, 53.0, 60.4, 71.9, 72.6, 78.5, 155.7, 172.3. MS: (ESI, pos): $\mathrm{m} / \mathrm{z}=338.2(\mathrm{M}+23)$. Anal. Calcd for $\mathrm{C}_{15} \mathrm{H}_{25} \mathrm{NO}_{6}$ : C, 57.13; H, 7.99; N, 4.44. Found: C, 56.89; H, 7.80; N, 4.47.

Ethyl diendo-(1S*,2S*,3R*,4R*)-3-(tert-butoxycarbonylamino)-5,6-dihydroxybicyclo[2.2.1]heptane-2carboxylate (5). White crystals; 88\% yield; m.p. 86-88 ${ }^{\circ} \mathrm{C} .{ }^{1} \mathrm{H}$ NMR (DMSO, $\left.400 \mathrm{MHz}\right) \delta$ : 1.13 $1.21\left(\mathrm{~m}, 4 \mathrm{H}, \mathrm{CH}_{3}\right.$ and $\left.\mathrm{H}-7\right), 1.38(\mathrm{~s}, 9 \mathrm{H}, t \mathrm{Bu}), 1.75(\mathrm{~d}, 1 \mathrm{H}, \mathrm{H}-7, J=10.32 \mathrm{~Hz}), 2.03-2.12(\mathrm{~m}, 1 \mathrm{H}$, H-4), 2.19-2.23 (m 1H, H-1), 2.90-2.99 (m, 1H, H-2), 3.81-4.1 (m, 5H, OCH, H-3, H-5 and H6), 4.45-4.63 (m, 2H, OH), $6.63(\mathrm{~d}, 1 \mathrm{H}, \mathrm{NH}, J=7.55 \mathrm{~Hz}) .{ }^{13} \mathrm{C}$ NMR (DMSO, $\left.400 \mathrm{MHz}\right) \delta: 14.9$, 29.0, 31.5, 44.2, 47.8, 49.0, 50.3, 60.8, 68.1, 69.5, 78.8, 156.0, 172.7. MS: (ESI, pos): $\mathrm{m} / \mathrm{z}=$ 339.1 (M+23). Anal. Calcd for $\mathrm{C}_{15} \mathrm{H}_{25} \mathrm{NO}_{6}$ : C, 57.13; H, 7.99; N, 4.44. Found: C, 57.09; H, 8.12; N, 4.57 . 
General procedure for cleavage of dihydroxy compounds and ring closure with reductive amination

Dihydroxy compound 2 or 5 (315 mg, $1 \mathrm{mmol})$ was dissolved in $\mathrm{THF} / \mathrm{H}_{2} \mathrm{O}(11 \mathrm{~mL}, 10: 1)$, and $\mathrm{NaIO}_{4}(0.42 \mathrm{~g}, 2 \mathrm{mmol})$ was added to the solution. After stirring for $1 \mathrm{~h}$ at $20{ }^{\circ} \mathrm{C}$ under an $\mathrm{Ar}$ atmosphere, $\mathrm{H}_{2} \mathrm{O}$ was added until the precipitation had dissolved. The mixture was extracted with $\mathrm{CH}_{2} \mathrm{Cl}_{2}(3 \times 40 \mathrm{~mL})$, the combined extract was dried over $\mathrm{Na}_{2} \mathrm{SO}_{4}$ and the resulting dialdehyde solution was immediately used for the next reaction without isolation. Benzylamine $(0.11 \mathrm{~mL}, 1 \mathrm{mmol})$ and oven-dried $3 \AA$ molecular sieve were added to the solution of the dialdehyde at $40{ }^{\circ} \mathrm{C}$, which was next stirred for $10 \mathrm{~min}$. A solution of $\mathrm{NaCNBH}_{3}(63 \mathrm{mg}, 1$ $\mathrm{mmol})$ and $\mathrm{AcOH}(0.057 \mathrm{~mL}, 1 \mathrm{mmol})$ in $\mathrm{EtOH}(2 \mathrm{~mL})$ was added dropwise during $2 \mathrm{~h}$ under an Ar atmosphere. The stirring was continued for another $1 \mathrm{~h}$ at $40{ }^{\circ} \mathrm{C}$. The reaction mixture was extracted in turn with $10 \% \mathrm{Na}_{2} \mathrm{CO}_{3}(3 \times 80 \mathrm{~mL})$, and brine $(80 \mathrm{~mL})$, dried over $\mathrm{Na}_{2} \mathrm{SO}_{4}$, and concentrated under reduced pressure. The residue was purified by column chromatography on silica gel, (3: $\mathrm{R}_{\mathrm{f}} 0.35 n$-hexane/EtOAc, 2:1; 6: $\mathrm{R}_{\mathrm{f}} 0.35 n$-hexane/EtOAc, 4:1).

Ethyl diexo-(1 $\left.R^{*}, 5 R^{*}, 6 S^{*}, 7 R^{*}\right)-3$-benzyl-7-(tert-butoxycarbonylamino)-3-azabicyclo[3.2.1]octane6-carboxylate (3). White crystals; 36\% yield (two steps); m.p. 100-103 ${ }^{\circ} \mathrm{C} .{ }^{1} \mathrm{H}$ NMR (DMSO, 400 $\mathrm{MHz}) \delta: 1.11-1.21\left(\mathrm{~m}, 4 \mathrm{H}, \mathrm{CH}_{3}\right.$ and $\left.\mathrm{H}-8\right), 1.36$ (s, 9H, tBu), 1.95-2.02 (m, 3H, H-2, H-4, H-1), 2.08-2.22 (m, 1H, H-8), 2.30-2.35 (m, 1H, H-5), 2.52-2.61 (m, 1H, H-4), 2.62-2.76 (m, 1H, H-2), $3.03(\mathrm{~d}, 1 \mathrm{H}, \mathrm{H}-6, J=8.31 \mathrm{~Hz}), 3.43\left(\mathrm{~s}, 2 \mathrm{H}, \mathrm{BnCH}_{2}\right), 3.80-3.95\left(\mathrm{~m}, 1 \mathrm{H}, \mathrm{OCH}_{2}\right), 3.96-4.09(\mathrm{~m}$, $\left.1 \mathrm{H}, \mathrm{OCH}_{2}\right), 4.22-4.26(\mathrm{~m}, 1 \mathrm{H}, \mathrm{H}-7), 6.76(\mathrm{~d}, 1 \mathrm{H}, \mathrm{NH}, J=9.06 \mathrm{~Hz}), 7.20-7.38(\mathrm{~m}, 5 \mathrm{H}, \mathrm{Ar}-\mathrm{H})$. ${ }^{13} \mathrm{C}$ NMR (DMSO, $\left.400 \mathrm{MHz}\right) \delta: 14.9,29.1,36.0,39.4,42.6,53.7,56.7,58.5,59.0,60.3,62.3$, 78.4, 127.7, 129.1, 129.4, 139.6, 155.6, 173.4. MS: (EI, pos): $\mathrm{m} / \mathrm{z}=388.5(\mathrm{M})$. Anal. Calcd for $\mathrm{C}_{22} \mathrm{H}_{32} \mathrm{~N}_{2} \mathrm{O}_{4}$ : C, 68.01; H, 8.30; N, 7.21. Found: C, 68.17; H, 8.15; N, 7.21.

Ethyl diendo-(1S*,5S*,6S*,7R*)-3-benzyl-7-(tert-butoxycarbonylamino)-3-azabicyclo[3.2.1]octane6-carboxylate (6). White crystals; 36\% yield; m.p. 78-80 ${ }^{\circ} \mathrm{C} .{ }^{1} \mathrm{H}$ NMR (DMSO, $\left.400 \mathrm{MHz}\right) \delta: 1.15$ (t, 3H, $\left.\mathrm{CH}_{3} J=7.08 \mathrm{~Hz}\right), 1.37(\mathrm{~s}, 9 \mathrm{H}, \mathrm{Bu}), 1.41-1.49$ (m, 1H, H-8), 1.52-1.61 (m, 1H, H-8), 1.97 (d, 1H, H-4, J = 10.93 Hz), 2.06-2.14 (m, 1H, H-1), 2.19 (d, 1H, H-2, J = 10.52 Hz), 2.24-2.32 (m, 1H, H-5), 2.53-2.57 (m, 1H, H-4), 3.06 (dd, 1H, H-6, $J_{1}=11.14$ Hz, $\left.J_{2}=5.70 \mathrm{~Hz}\right), 3.17-3.27$ $\left(\mathrm{m}, 2 \mathrm{H}, \mathrm{BnCH}_{2}\right.$, and $\left.\mathrm{H}-2\right), 3.50\left(\mathrm{~d}, 1 \mathrm{H}\right.$ and $\left.\mathrm{BnCH}_{2}, J=12.56 \mathrm{~Hz}\right), 4.03\left(\mathrm{~m}, 2 \mathrm{H}, \mathrm{OCH}_{2}\right), 4.15-$ $4.27(\mathrm{~m}, 1 \mathrm{H}, \mathrm{H}), 6.04(\mathrm{~d}, 1 \mathrm{H}, \mathrm{NH}, J=9.92 \mathrm{~Hz}), 7.18-7.35$ (m, 5H, Ar-H). ${ }^{13} \mathrm{C}$ NMR (DMSO, $400 \mathrm{MHz}) \delta: 14.9,29.0,35.9,38.0,47.2,51.6,54.8,56.9,60.2$, 62.8, 78.3, 127,8, 129.1, 129.6, 139.2, 156.0, 171.9. MS: (ESI, pos): $\mathrm{m} / \mathrm{z}=389.3(\mathrm{M}+1)$. Anal. Calcd for $\mathrm{C}_{22} \mathrm{H}_{32} \mathrm{~N}_{2} \mathrm{O}_{4}$ : C, 68.01; H, 8.30; N, 7.21. Found: C, 67.88; H, 8.49; N, 7.11.

\section{Isomerization of amino ester 6}

Freshly prepared NaOEt (57 mg, $0.83 \mathrm{mmol})$ was added to a solution of 6 (250 $\mathrm{mg}, 0.64 \mathrm{mmol})$ in anhydrous EtOH $(5 \mathrm{~mL})$, and the mixture was stirred at room temperature for $48 \mathrm{~h}$. The reaction mixture was then concentrated under reduced pressure and taken up in EtOAc $(15 \mathrm{~mL})$, and the organic layer was washed with $\mathrm{H}_{2} \mathrm{O}(3 \times 5 \mathrm{~mL})$. The combined extract was dried over 
$\mathrm{Na}_{2} \mathrm{SO}_{4}$ and concentrated under reduced pressure. The residue was purified by column chromatography on silica gel ( $n$-hexane/EtOAc, $3: 1)$.

Ethyl $\left(1 S^{*}, 5 S^{*}, 6 R^{*}, 7 R^{*}\right)-3$-benzyl-7-(tert-butoxycarbonylamino)-3-azabicyclo[3.2.1]octane6-carboxylate (7). Colorless oil; 77\% yield. ${ }^{1} \mathrm{H}$ NMR (DMSO, $\left.400 \mathrm{MHz}\right) \delta: 1.17$ (t, 3H, $\mathrm{CH}_{3}, J$ $=7.08 \mathrm{~Hz}), 1.32(\mathrm{~s}, 9 \mathrm{H}, t \mathrm{Bu}), 1.36-1.39(\mathrm{~m}, 1 \mathrm{H}, \mathrm{H}-8), 1.62-1.72(\mathrm{~m}, 1 \mathrm{H}, \mathrm{H}-8), 1.96-1.99(\mathrm{~m}, 1 \mathrm{H}$, $\mathrm{H}-2)$, 2.07-2.27 (m, 3H, H-5, H-4 and H-1), 2.52-2.83 (m, 3H, H-2, H-4 and H-6), 3.35 (d, 1H, $\left.\mathrm{BnCH}_{2}, J=12.2 \mathrm{~Hz}\right), 3.54\left(\mathrm{~d}, 1 \mathrm{H}, \mathrm{BnCH}_{2}, J=12.2 \mathrm{~Hz}\right), 3.98-4.14\left(\mathrm{~m}, 3 \mathrm{H}, \mathrm{OCH}_{2}\right.$ and $\left.\mathrm{H}-7\right), 5.87$ $(\mathrm{d}, 1 \mathrm{H}, \mathrm{NH}, J=8.08 \mathrm{~Hz}), 7.20-7.39$ (m, 5H, Ar-H). ${ }^{13} \mathrm{C}$ NMR (DMSO, $\left.400 \mathrm{MHz}\right) \delta: 13.4,27.7$, 34.1, 37.0, 38.2, 52.3, 55.2, 58.7, 59.5, 60.9, 78.0, 126.6, 128.1, 128.8, 138.0, 154.8, 174.6. Anal. Calcd for $\mathrm{C}_{22} \mathrm{H}_{32} \mathrm{~N}_{2} \mathrm{O}_{4}$ : C, 68.01; H, 8.30; N, 7.21. Found: C, 68.02; H, 8.46; N, 7.20.

\section{Characterization of enantiomeric compounds}

All the reactions were first optimized for racemic compounds. The ${ }^{1} \mathrm{H}$ and ${ }^{13} \mathrm{C}$ NMR spectroscopic data and elemental analyses on the enantiomeric derivatives were in accordance with those for the racemic compounds. Representative data on the enantiomers are as follows:

Ethyl (1S,2S,3R,4R)-3-(tert-butoxycarbonylamino)bicyclo[2.2.1]hept-5-ene-2-carboxylate $[(+)-1]$. White crystals; 99\% yield; $[\alpha]_{\mathrm{D}}:+90$ (c 0.245, EtOH); m.p. 101-103 ${ }^{\circ} \mathrm{C}$.

Ethyl (1R,2S,3R,4S)-3-(tert-butoxycarbonylamino)-5,6-dihydroxybicyclo[2.2.1]heptane-2carboxylate $[(+)-2]$. Yellow oil; 98\% yield; $[\alpha]_{\mathrm{D}}:+41$ (c 0,245, EtOH).

Ethyl (1R,5R,6S,7R)-3-benzyl-7-(tert-butoxycarbonylamino)-3-azabicyclo[3.2.1]octane-6carboxylate $[(+)-3]$. White crystals; 38\% yield; $[\alpha]_{D}:+53($ c 0,245, EtOH); ee $>99$ \%; m.p. 80$82{ }^{\circ} \mathrm{C}$.

\section{Acknowledgements}

The authors are grateful to the Hungarian Research Foundation (OTKA No F67970 and NK81371) for financial support and for the award of Bolyai János Fellowships to Loránd Kiss and Enikő Forró.

\section{References}

1. (a) Kiss, L.; Forró, E.; Fülöp, F. Synthesis of carbocyclic $\beta$-amino acids. Amino Acids, Peptides and Proteins in Organic Chemistry. Vol. 1, Hughes, A. B., Ed. Wiley: Weinheim, 2009, 367. (b) Fülöp, F. Chem. Rev. 2001, 101, 2181. (c) Mittendorf, J.; Kunisch, F.; Matzke, M.; Militzer, H-C.; Schmidt, A.; Schönfeld, W. Bioorg. Med. Chem. Lett. 2003, 13, 433. (d) Yang, D.; Zhang, D-W.; Hao, Y.; Wu, Y-D.; Luo, S-W.; Zhu, N-Y. Angew. Chem. Int. Ed. 2004, 43, 6719. (e) Rathore, N.; Gellman, S. H.; Pablo, J. J. Biophys. J. 2006, 91, 3425. (f) Porter, E. A.; Weisblum, B.; Gellman, S. H. J. Am. Chem. Soc. 2005, 127, 11516. (g) Roy, 
O.; Faure, S.; Aitken, D. J. Tetrahedron Lett. 2006, 47, 5981. (h) Chandrasekhar, S.; Sudhakar, A.; Kiran, M. U.; Babu, B. N.; Jagadeesh, B. Tetrahedron Lett. 2008, 49, 7368. (i) Rua, F.; Boussert, S.; Parella, T.; Diez-Perez, I.; Branchadell, V.; Giralt, E.; Ortuno, R. M. Org. Lett. 2007, 9, 3643. (j) D’Elia, V.; Zwicknagl, H.; Reiser, O. J. Org. Chem. 2008, 73, 3262. (k) Fülöp, F.; Martinek, T. A.; Tóth, G. K. Chem. Soc. Rev. 2006, 35, 323. (1) Hetényi, A.; Mándity, I. M.; Martinek, T. A.; Tóth, G. K.; Fülöp, F. J. Am. Chem. Soc. 2005, 127, 547. (m) Torres, E.; Acosta-Silva, C.; Rua, F.; Alvarez-Larena, A.; Parella, T.; Branchadell, V.; Ortuno, R. M. Tetrahedron 2009, 65, 5669. (n) Fernandez, D.; Torres, E.; Aviles, F. X.; Ortuno, R. M.; Vendrell, J. Bioorg. Med. Chem. 2009, 17, 3824. (o) Fernandes, C.; Pereira, E.; Faure, S.; Aitken, D. J. J. Org. Chem. 2009, 74, 3217.

2. (a) Kiss, L.; Kazi, B.; Forró, E.; Fülöp, F. Tetrahedron Lett. 2008, 49, 339. (b) Kazi, B.; Kiss, L.; Forró, E.; Fülöp, F. Tetrahedron Lett. 2010, 51, 82 and references cited herein. (c) Porter, E. A.; Wang, X.; Lee, H-S.; Weisblum, B.; Gellman, S. H. Nature 2000, 404, 565. (d) Porter, E. A.; Weisblum, B.; Gellman, S. H. J. Am. Chem. Soc. 2005, 127, 11516. (e) Wang, G. T.; Chen, Y.; Wang, S.; Gentles, R.; Sowin, T.; Kati, W.; Muchmore, S.; Giranda, V.; Stewart, K.; Sham, H.; Kempf, D.; Laver, W. G. J. Med. Chem. 2001, 44, 1192. (f) Brown, J. R.; Nishimura, Y., Esko, J. D. Bioorg. Med. Chem. Lett. 2006, 16, 532. (g) Ott, G. R.; Asakawa, N.; Lu, Z.; Liu, R. Q.; Covington, M. B.; Vaddi, K.; Qian, M.; Newton, R. C.; Christ, D. D.; Traskos, J. M.; Decicco, C. P.; Duan, J. J. W. Bioorg. Med. Chem. Lett. 2008, 18, 694.

3. (a) Avenoza, A.; Barriobero, J. I.; Busto, J. H.; Cativiela, C.; Peregrina, J. M. Tetrahedron: Asymmetry 2002, 13, 625. (b) Gil, A. M.; Bunuel, E.; Lopez, P.; Cativiela, C. Tetrahedron: Asymmetry 2004, 15, 811.

4. (a) Casabona, D.; Jimenez, A. I.; Cativiela, C. Tetrahedron 2007, 63, 5056. (b) Demizu, Y.; Shiigi, H.; Mori, H.; Matsumoto, K.; Onomura, O. Tetrahedron: Asymmetry 2008, 19, 2659.

5. (a) Otani, Y.; Futaki, S.; Kiwada, T.; Sugiura, Y.; Muranaka, A.; Kobayashi, N.; Uchiyama, M.; Yamaguchi, K.; Ohwada, T. Tetrahedron 2006, 62, 11635. (b) Pandey, G.; Laha, J. K.; Lakshmaiah, G. Tetrahedron 2002, 58, 3525.

6. Armstrong, A.; Bhonoah, Y.; White, A. J. P. J. Org. Chem. 2009, 74, 5041.

7. (a) Parsons, P. J.; Camp, N. P.; Edwards, N.; Sumoreeah, L. R. Tetrahedron 2000, 56, 309

(b) Brenneman, J. B.; Machauer, R.; Martin, S. F. Tetrahedron 2004, 60, 7301. (c) Marc, M.; Outurquin, F.; Renard, P-Y.; Créminon, C.; Franck, X. Tetrahedron Lett. 2009, 50, 4554.

8. (a) Soriano, E.; Contelles, J. M. J. Org. Chem. 2009, 74, 4061. (b) Armstrong, A.; Bhonoah, Y.; Shanahan, S. E. J. Org. Chem. 2007, 72, 8019. (c) Chen, Z. M.; Trudell, M. L. Chem. Rev. 1996, 96, 1179. (d) Aggarwal, V. K.; Olofsson, B. Angew. Chem. Int. Ed. 2005, 44, 5516. (e) Daly, j. W. J. Med. Chem. 2003, 46, 445. (f) Runyon, S. P.; Burgess, J. P.; Abraham, P.; Keverline-Franz, K. I.; Flippen-Anderson, J.; Dechamps, J.; Lewin, A. H.; Navarro, H. A.; Boja, J. W.; Kuhar, M. J.; Carroll, F. I. Bioorg. Med. Chem. 2005, 13, 2439. (g) Jin, C.; Navarro, H. A.; Carroll, F. I. Bioorg. Med. Chem. 2009, 17, 5126.

9. (a) Gelmi, M. L.; Cattaneo, C.; Pellegrino, S.; Clerici, F.; Montali, M.; Martini, C. J. Org. Chem. 2007, 72, 9811 and references cited herein. (b) Caputo, F.; Cattaneo, C.; Clerici, F.; 
Gelmi, M. L.; Pellegrino, S. J. Org. Chem. 2006, 71, 8467. (c) Brocke C.; Brimble, M. A.; Lin, D, S-H.; McLeod, M. D. Synlett 2004, 2359. (d) Buckley, B. R.; Page, P. C. B.; Heaney, H.; Sampler, E. P.; Carley, S.; Brocke, C.; Brimble, M. A. Tetrahedron 2005, 61, 5876. (e) Yamashita, A.; Takahashi, N.; Mochizuki, D.; Tsujita, Yamada, S.; Kawakubo, H.; Suzuki, Y.; Watanabe, H. Bioorg. Med. Chem. Lett. 1997, 7, 2303. (f) Lew, W.; Wu, H.; Chen, X.; Graves, B. J.; Escarpe, P. A.; MacArthur, H. L.; Mendel, D. B.; Kim. C. U. Bioorg. Med. Chem. 2000, 10, 1257.

10. (a) Stájer, G.; Szabó, E. A.; Bernáth, G.; Sohár, P. J. Chem. Soc., Perkin Trans. 1 1987, 237.

(b) Stájer, G.; Szabó, E. A.; Bernáth, G.; Fülöp, F.; Sohár, P. Chemische Berichte 1987, 120, 259. (c) Stájer, G.; Szabó, E. A.; Fülöp, F.; Bernáth, G.; Sohár, P. J. Heterocyclic Chem. 1983, 20, 1181.

11. Forró, E.; Fülöp, F. Tetrahedron: Asymmetry 2004, 15, 573. 